\title{
Identified mutations in CYP11B1 gene in two Tunisian patients with 11- beta hydroxylase deficiency
}

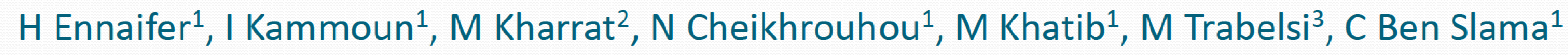 \\ 1-Department of endocrinology and metabolic diseases, National Institute of Nutrition, Tunis, Tunisia \\ 2-Laboratory of Human Genetics, University of medicine of Tunis, Tunisia \\ 3-Department of congenital and hereditary diseases, Charles-Nicolle Hospital, Tunis, Tunisia
}

\section{Introduction:}

11-Beta hydroxylase deficiency (11-OHD), a rare autosomal recessive disorder, is caused by $\mathrm{CYP} 11 \mathrm{~B} 1$ mutations. The incidence of $11-\mathrm{OHD}$ in overall population is approximately 1 in $100,000-200,000$ [1]. Based on clinical manifestations, 11-OHD is classified as classic and non-classic forms.

We studied the mutations of $C Y P_{11 B}$ gene in two patients with classic $11 \beta-O H D$.

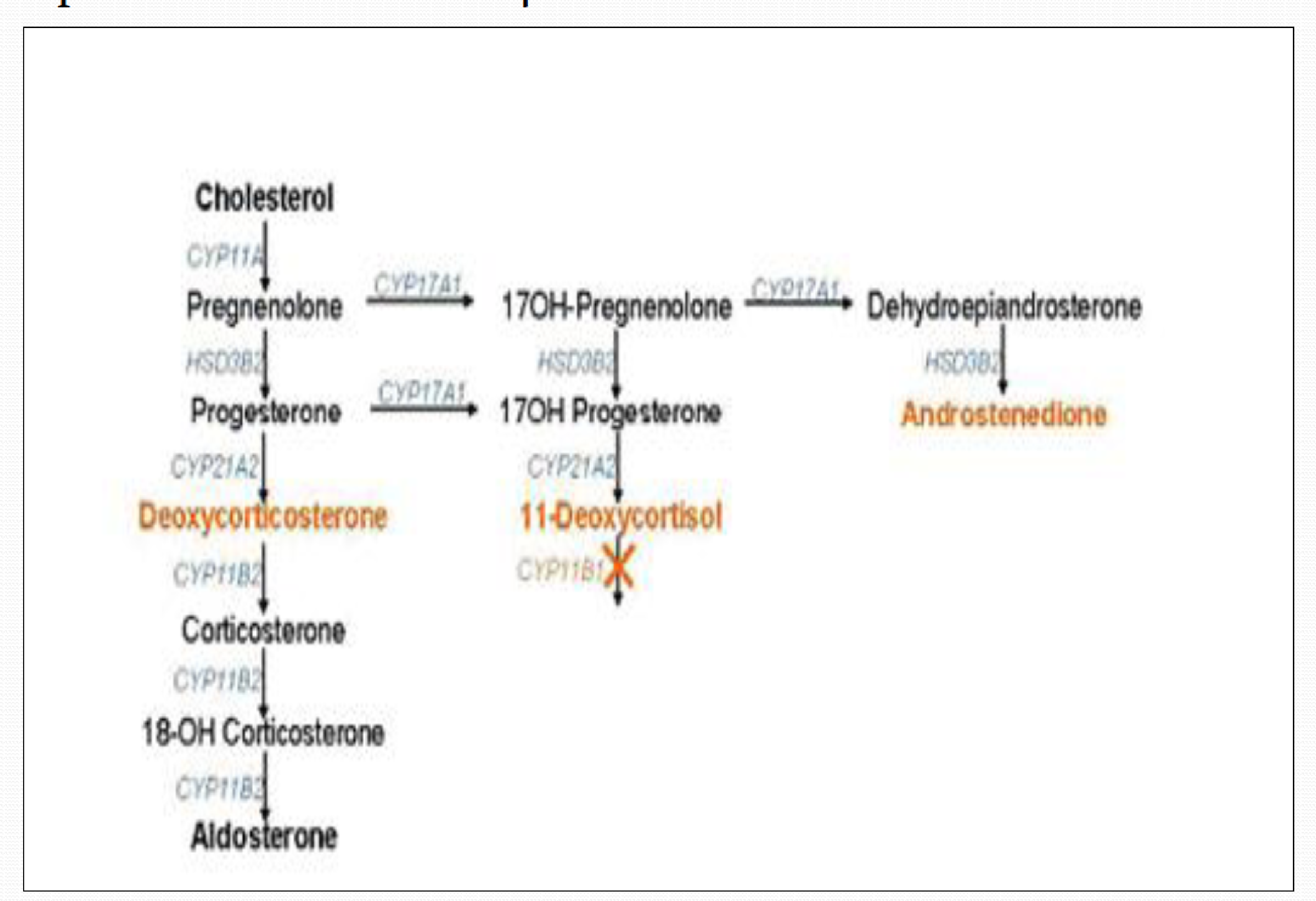

Figure 1: 11Beta-Hydrolyase Deficiency Classic Form

Observations:

We present the first case of 23 years old boy with preliminary diagnosis of $21 \beta$-OHD diagnosed at the age of 10 years. The patient presented with hypertension and hypokalemia which were against the diagnosis of 21 $\beta$-OHD. The examination of the external genitalia shows a micropenis and an empty testicular purse. The patient's karyotype was $46 \mathrm{XX}$. Cortisol level was normal. Deoxycorticosterone, corticosterone, DHEA and $17 \mathrm{OH}$-progesterone were markedly elevated. The hypothesis of $11 \beta-O H D$ deficiency was considered and confirmed by genetic exploration. A non-sense mutation $6379 \mathrm{~V}$ of the CYP11 gene was found. The patient was forwarded to an experienced surgeon for micropenis.

The second case is about a 9 years old girl who was diagnosed at birth with a genital ambiguity and the karyotype was $46 \mathrm{XX}$. The patient had a feminizing surgery at the age of 6 months. The patient developed a hypertension at the age of 6 and presented with severe hypokalemia. Deoxycorticosterone and ${ }_{17} \mathrm{OH}-$ progesterone were markedly elevated.
The hypothesis of $11 \beta-O H D$ deficiency was considered and confirmed by genetic exploration. A non-sense mutation p.G379V of the CYP11Bı gene was found.

\section{Discussion:}

In classic $11-O H D$, a reduction of adrenal cortisol synthesis leads to elevated plasma ACTH levels, which result in an increased production of cortisol precursors in zona fasciculate. Therefore, the androgen synthesis is increased and hyperandrogenemia appears. Moreover, the increased ACTH secretion also contributes to higher levels of deoxycortisone (DOC) and 11deoxycortisol [2]. Thus, classic $11-\mathrm{OHD}$ is characterized by severe virilization in newborn females and precocious pseudopuberty in both sexes. The DOC overproduction causes hypertension and hypokalemia.

Non-classic 11-OHD is usually characterized by slight increase of serum androgen, mild hirsutism and irregular menses. However, elevated blood pressure is rarely observed in the mild form. CYP11B1 gene consists of nine exons and encodes a protein of 503 amino acids. CYP11B1 gene is located on chromosome 8q22, approximately $40 \mathrm{~kb}$ apart from the aldosterone synthase gene (CYP11B2) [3]. Todate, more than 50 mutations have been reported in patients with $11-\mathrm{OHD}$, which are clustered in exons 2 , 6-8 [4].

The first case is particularly interesting because of the delay of diagnosis. The $11 \beta$-hydroxylase deficiency diagnosis is to be considered when hypertension is associated with hypokalemia and hypogonadism, even in adult patients. A particular challenge in the diagnosis of classic $11 \mathrm{OHD}$ is the lack of standardized diagnostic endocrine work-up; thus, rare and untypical cases are likely to be missed. Early diagnosis and start of disease-specific treatment is important to avoid severe long-term consequences such as hyperandrogenism and potentially hypertension.

The second case shows the relationship between surgical outcome and psychological development. Severely virilized cases may initially be assigned as males, and once such assignment has been made, it may be difficult to reverse, thereby sacrificing fertility. Early medical, psychological and surgical treatment of children with virilizing congenital adrenal hyperplasia should enable them to become normal adults[5].

\section{References:}

[1] P.W. Speiser, P.C. White, J. Dupont, D. Zhu, A.B. Mercado, M.I. New, Prenatal diagnosis of congenital adrenal hyperplasia due to 21-hydroxylase deficiency by allele-specific hybridization and Southern blot, Human Genetics 93 (1994) 424-428.

[2] P.W. Speiser, P.C. White, Congenital adrenal hyperplasia, The New England Journal of Medicine 349 (2003) $776-788$.

[3] E. Mornet, J. Dupont, A. Vitek, P.C. White, Characterization of two genes encoding human steroid 11 beta-hydroxylase (P-45o(11) beta), Journal of Biological Chemistry 264 (1989) 20961-20967.

[4] N. Krone, W. Arlt, Genetic of congenital adrenal hyperplasia, Best Practice and Research: Clinical Endocrinology and Metabolism 23 (2009) 181-192.

[5] Bistritzer T, Sack J, Eshkol A, Zur H, Katznelson D. Sex reassignment in a girl with 11 beta-hydroxylase deficiency. Isr J Med Sci. 1984 Jan;20(1):55-8 